\title{
Probabilidad de lesión de tren inferior e índice de grasa corporal en talleres deportivos universitarios
}

\section{Probability of lower body injury and body fat index in university sports workshops}

\author{
*Fredy Cáceres Montecinos, **Vanessa Carrasco Alarcón, \\ **Cristian Martínez Salazar
}

Cáceres, F., Carrasco, V., \& Martínez, C. (2020). Probabilidad de lesión de tren inferior e índice de grasa corporal en talleres deportivos universitarios. Revista Ciencias de la Actividad Física UCM, N²1(1), enero-junio, 1-10. DOI: http://doi.org/10.29035/rcaf.21.1.6

\section{RESUMEN}

El objetivo es verificar si existe relación entre la probabilidad de lesión en extremidades del tren inferior y el índice de grasa corporal en estudiantes que participan de talleres deportivos en la Universidad Adventista de Chile. Participaron 66 alumnos entre las áreas deportivas vóleibol, básquetbol, fútbol y hándbol por un método de muestreo censal. Todos los participantes de la investigación fueron evaluados por medio del instrumento "Y balance Test", para verificar si son propensos a sufrir una lesión de tren inferior y además se les realizó una evaluación antropométrica para conocer su índice de grasa corporal. Los resultados no encontraron correlación entre porcentaje de grasa y probabilidad de lesión, sin embargo, se encontró correlación entre probabilidad de lesión entre una pierna y otra.

\section{Palabras clave}

Lesión, Y balance test, talleres deportivos, estudiantes universitarios.

\begin{abstract}
The objective is to verify if there is a relationship between the likelihood of limb injury in the lower body and the body fat index in students participating in sports workshops at the Adventist University of Chile. 66 students participated in sports areas such as volleyball, basketball, soccer and handball by a census sampling method. All the participants of the investigation were evaluated by means of the "Y balance Test" instrument, to verify if they are prone to suffer a lower train injury and also an anthropometric evaluation was done to know their body fat index. The results did not find correlation between fat percentage and injury probability, however, a correlation was found between the injury probability between one leg and the another.
\end{abstract}

Key words

Injury, Y balance test, sports workshops, university students.

* Universidad Adventista de Chile. Chillán, Chile.

** Universidad de La Frontera. Temuco, Chile. 
Cáceres, F., Carrasco, V., \& Martínez, C. (2020). Probabilidad de lesión de tren inferior e índice de grasa corporal en talleres deportivos universitarios. Revista Ciencias de la Actividad Física UCM, N 21(1), enero-junio, 1-10. DOI: http://doi.org/10.29035/rcaf.21.1.6

\section{Introducción}

Una de las opciones que ofrece la mayoría de las universidades al alumnado son los talleres deportivos, en los cuales, como mencionan los autores Warburton, Nicol, y Bredin, (2006) brindan numerosos beneficios al participante, entre ellos: reducir el riesgo de desarrollar enfermedades cardiovasculares, diabetes u osteoporosis, entre otras. Sin embargo, el deporte tiene asociado un riesgo intrínseco de lesión al participante. Meeuwisse, Tyreman, Hagel y Emery (2007) se refieren a que una lesión ocurre cuando la cantidad de energía mecánica que se transfiere al cuerpo excede el umbral humano, provocando daño tisular, es decir, la fuerza recibida es mayor a la fuerza que resiste un tejido. Así también, Chimera, Smith y Warren, (2015) explican en su estudio sobre "historial de lesiones" que las lesiones músculo-esqueléticas son un riesgo inherente dentro de la práctica deportiva, de modo que el riesgo de sufrir una lesión sin contacto representan aproximadamente el $20 \%$ de todas las lesiones durante los juegos y el $40 \%$ de las lesiones sufridas durante las prácticas, Boechat de Oliveira et al., (2017) también hacen referencia a esta idea sobre lesiones durante los entrenamientos en su estudio sobre lesiones en motocross, indicando que una gran parte de las lesiones ocurren fuera del campo de la competencia. De igual forma Andrade y Villena (2006) refieren a que la lesión más frecuente en deportistas implica la articulación de tobillo, la cual tiene una frecuencia del 20\% y constituye un 15\% de las emergencias asociadas al deporte. Los deportistas que más sufren lesiones de este tipo son los jugadores de fútbol con un $25 \%$ y los jugadores de básquetbol con un $40 \%$.

La composición corporal es un factor que puede influir directamente a la mayoría de la población, sobre todo a los deportistas en cuanto al índice lesional (Kenney, Wilmore,
Costill, y Wilmore, 2012). Generalmente se menciona al IMC en lugar del porcentaje de grasa, sin embargo se ha determinado que factores como la grasa, la circunferencia abdominal y también el sobrepeso indicado desde el porcentaje de masa grasa, son mejores indicadores de riesgo de lesión y estado de salud que el IMC (Medina, Lizarraga, y Drobnic, 2014). Así también Amaral et al. (2011) mencionaron que uno de los posibles factores de riesgo de lesión se constituye por el exceso de grasa corporal, el cual en algunos casos es un problema importante en la salud. Por consiguiente, la composición corporal ideal varía entre los deportes; en general, cuanto menor es el contenido de grasa, mejor es el rendimiento, sin embargo, la natación puede ser una excepción, así lo indican Martínez et al. (2011). En este sentido, generalmente los estudiantes universitarios no cuentan con característícas físicas óptimas para el deporte que practican, tal como describe Rivera (2002) quien al analizar distintos deportes encontró una tendencia hacia el tipo corporal endomorfo por sobre el mesomorfo, el cual, es el somatotipo de referencia de un atleta profesional, así mismo Padilla, Taylor, Yuhasz y Velazquez (2004) en un estudio similar, concluyen que el somatotipo de los atletas estudiantes de la Ciudad de México en su mayoría es endomorfo-balanceado resultando ser diferente respecto de los atletas de nivel olímpico (mesomorfo balanceado).

Dentro de los factores de riesgo de lesión que mencionan Osorio, Clavijo, Arango, Patiño y Gallego (2007) se encuentra la composición corporal, dentro de la cual, se destaca el peso corporal que genera aumento de la carga y tiene impacto sobre las articulaciones, por lo tanto, se puede inferir que a mayor porcentaje de grasa corporal, el participante tendrá más peso, por consecuencia, puede tener un 
mayor riesgo de lesión. Stevenson, Hamer, Finch, Elliot y Kresnow (2000) refieren que en general, las lesiones deportivas son más comunes en programas recreativos, deportistas en formación o aficionados, que en deportistas profesionales.

Por otro lado Plisky et al. (2009) explican dentro de su estudio, que uno de los métodos que se emplea para predecir la probabilidad de lesión, es el test de excursión en estrella (SEBT) el cual ha sido utilizado para medir el rendimiento físico, comparar la capacidad de equilibrio entre diferentes deportes, e identificar individuos que tienen inestabilidad crónica del tobillo, últimamente utilizado para identificar a los atletas con mayor riesgo de lesión. Asimismo, el Y Balance Test (YBT) es una prueba confiable, correspondiente a una versión desarrollada para mejorar la repetibilidad de medición y estandarizar el rendimiento de la prueba SEBT. Al mismo tiempo, para llevar un control sobre el índice de grasa corporal existen diversos métodos, entre ellos el cáliper, el cual ha conseguido gran protagonismo en materia de antropometría. Según Martín, Ross, Drinkwater, y Clarys (1992) este ha sido considerado como el instrumento doblemente indirecto más razonable al momento de obtener datos. Además del cáliper, existe el método de bioimpedancia eléctrica, del cual, destacan atributos como: masivo, económico, simple y rápido al momento de su uso (Alvero, Correas, Ronconi, Fernández, \& Porta, 2011).

Finalmente, es fundamental realizar controles de prevención sobre posibles lesiones en el deporte a nivel universitario y establecer si existe relación entre estas y el índice de grasa corporal, de ser así, se deben emplear rutinas de entrenamiento para controlar el nivel de grasa corporal, fortalecer las extremidades inferiores para que se encuentren preparadas ante posibles impactos inesperados o movimientos que sobre exijan zonas del cuerpo.

\section{Método}

La investigación presenta un diseño analítico, transversal. La muestra es de carácter censal, y correspondió a 66 alumnos, de ambos sexos, matriculados en la Universidad Adventista de Chile, participantes de los talleres deportivos: fútbol, vóleibol, básquetbol y hándbol. Entre los criterios de inclusión se solicitó cumplir con requisitos de edad (18-35 años), que realicen entrenamiento dos veces a la semana (dentro o fuera del taller), que estén inscritos formalmente en el taller, y que practiquen el deporte con un mínimo de 3 meses de antelación. Así también dentro de los criterios de exclusión se omitieron participantes que no quisieron participar de la investigación o que no hayan firmado el consentimiento informado.

Para evaluar la probabilidad de lesión de tren inferior se realizó la prueba de equilibrio mediante el instrumento "Y balance test" (YBT), el cual proviene de la prueba de equilibrio de excursión estrella (SEBT) (Chimera, Smith y Warren, 2015) presentando validez en el estudio de Llacer (2015) y de Gribble, Hertel, \& Plisky (2012). El Kit YBT consiste en una plataforma a la cual se adjuntan 3 tubos de PVC en su cara anterior, posteromedial y posterolateral. Cada tubo se encuentra marcado con líneas de 5 milímetros de diferencia, en el cual el participante empuja con un pie el indicador de alcance unido al tubo milimetrado, mientras realiza equilibrio con el otro pie. Debe empujar en las 3 direcciones. Al expresar la distancia de alcance como un porcentaje de la longitud de la extremidad, el valor normalizado se calcula como la distancia de alcance dividida por la longitud de la extremidad, consecutivamente el resultado anterior se multiplica por 100. Así también una diferencia de alcance anterior de $4 \mathrm{~cm}$ o más o un puntaje compuesto menor o igual al 94\% de la longitud de 
la pierna, es un predictor de lesión en la extremidad inferior (Plisky et al., 2009).

Por otro lado, para conocer el porcentaje de grasa corporal se comenzó utilizando la fórmula de durnin/womersley para obtener Densidad Corporal (DC) (Moreno, Gòmez Gandoy, y Antoranz González, 2001) por medio de la medición de pliegues cutáneos, tales como, bicipital, tricipital, sub escapular y supraespinal según el protocolo ISAK (Stewart, Marfell-Jones, Olds, y de Ridder, 2011) utilizando un cáliper Slim Guide (Hewitt, Withers, Brooks, Woods, y Day, 2002).

Los datos obtenidos para cada variable fueron analizados en Software Estadístico para las ciencias sociales (SPSS), en su vigésima versión para Windows. Se hizo una descripción de las variables cuantitativas mediante medias y desviación estándar, y de las variables cualitativas mediante tablas de frecuencia y porcentajes. Para el desarrollo de la estadística analítica se utilizó la prueba de Kolmogorov-Smirnov para determinar la normalidad de las variables, posteriormente se utilizaron pruebas paramétricas para establecer la relación entre probabilidad de lesión y porcentaje de grasa corporal según sexo según Prueba t; y para establecer la relación entre las variables Probabilidad Lesión de Pierna derecha, Probabilidad Lesión de Pierna izquierda y Porcentaje de Grasa utilizando la correlación de Pearson. Frente a los resultados obtenidos en la correlación se realizó un gráfico de dispersión de las variables Probabilidad Lesión en Pierna derecha e izquierda.

\section{Resultados}

Esta investigación se realizó sobre un total de 66 alumnos universitarios (41 varones y 25 damas) participantes en talleres deportivos como fútbol (16), vóleibol (19), básquetbol (16) y hándbol (15) con una edad media de 21 de años. De la totalidad de la muestra 9 participantes utilizan su pierna izquierda como dominante (hábil), frente a los otros 57 que utilizan su pierna derecha. En la tabla 1 se puede apreciar los resultados en cuanto a probabilidad de lesión de pierna izquierda y derecha, y así también el porcentaje de grasa corporal en damas y varones.

Tabla 1

Prueba t para comparar medias de probabilidad de lesión y porcentaje de grasa corporal según sexo.

\begin{tabular}{|c|c|c|c|c|c|c|c|}
\hline & Sexo & $N$ & Media & $\begin{array}{l}\text { Desviación } \\
\text { Tip. }\end{array}$ & $\begin{array}{l}\text { Error tip. de } \\
\text { la media }\end{array}$ & $\mathrm{T}$ & $P$ \\
\hline $\begin{array}{l}\text { Probabilidad } \\
\text { de Lesión } \\
\text { pierna derecha }\end{array}$ & $\begin{array}{l}\text { Masculino } \\
\text { Femenino }\end{array}$ & $\begin{array}{l}4 \\
1 \\
2 \\
5\end{array}$ & $\begin{array}{l}98,89 \\
998,49 \\
7\end{array}$ & $\begin{array}{l}6,840 \\
5,785\end{array}$ & $\begin{array}{l}1,06 \\
8 \\
1,157\end{array}$ & 251 & ,803 \\
\hline $\begin{array}{l}\text { Probabilidad } \\
\text { de Lesión } \\
\text { pierna } \\
\text { izquierda }\end{array}$ & $\begin{array}{l}\text { Masculino } \\
\text { Femenino }\end{array}$ & $\begin{array}{l}4 \\
1 \\
2 \\
5 \\
\end{array}$ & $\begin{array}{l}99,63 \\
9 \\
97,81 \\
6 \\
\end{array}$ & $\begin{array}{l}6,287 \\
4,756\end{array}$ & $\begin{array}{l}, 981 \\
, 951\end{array}$ & 1,247 & ,217 \\
\hline $\begin{array}{l}\text { Porcentaje de } \\
\text { Grasa }\end{array}$ & $\begin{array}{l}\text { Masculino } \\
\text { Femenino }\end{array}$ & $\begin{array}{l}4 \\
1 \\
2 \\
5\end{array}$ & $\begin{array}{l}15,95 \\
1 \\
28,59 \\
4\end{array}$ & $\begin{array}{l}5,819 \\
4,288\end{array}$ & $\begin{array}{l}, 908 \\
, 857\end{array}$ & 9,405 & ,000 \\
\hline
\end{tabular}


La prueba de correlación de Pearson no indicó relación entre la probabilidad de lesión de la pierna derecha y el porcentaje de grasa corporal de los estudiantes $(p>0,05)$, mientras que la probabilidad de lesión de la pierna izquierda frente al porcentaje de grasa corporal presenta una débil correlación inversamente proporcional, $(p=0,049 r=-0,243)$ lo que indica que, a mayor probabilidad de lesión de la pierna izquierda, existe un menor porcentaje de grasa corporal. Tal y como se demuestra a continuación en la Tabla 2.

Tabla 2

Correlación para las variables probabilidad de lesión y porcentaje de grasa.

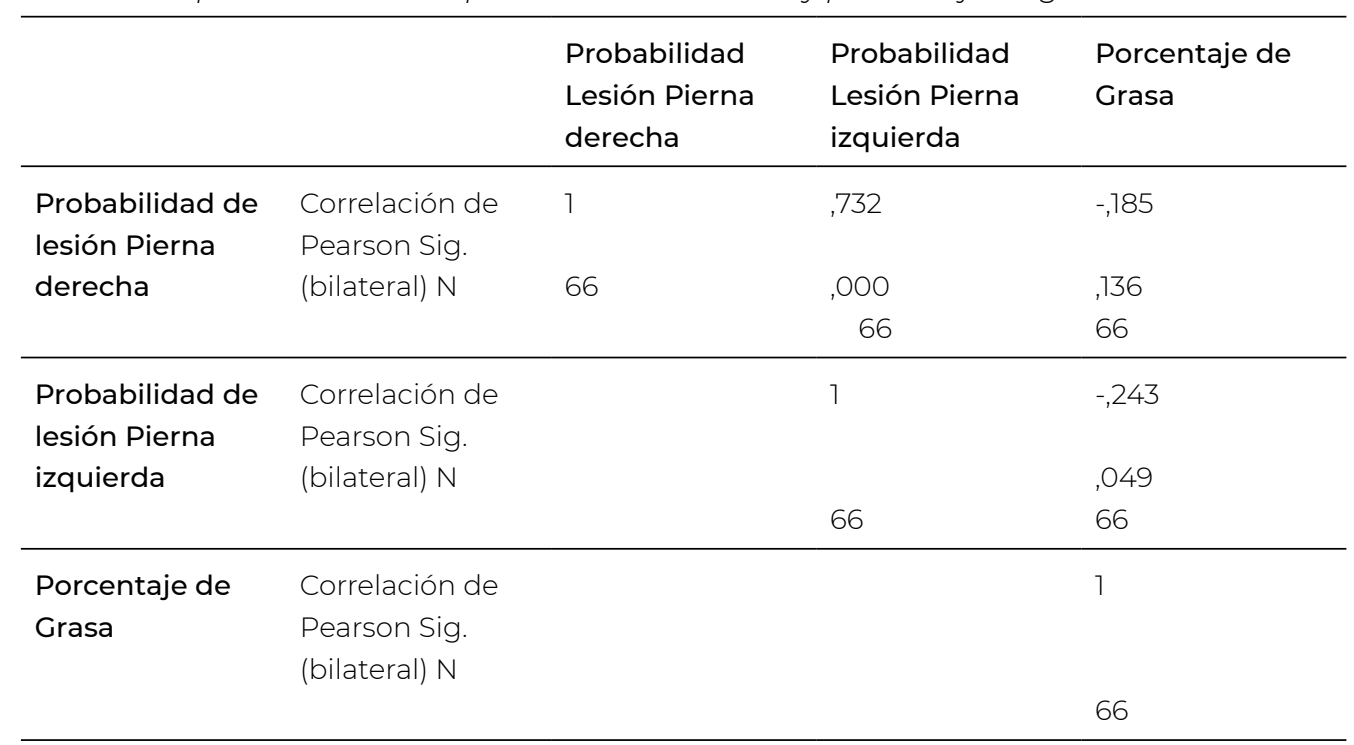

Por otro lado, según el modelo de correlación de Pearson, la probabilidad de lesión en la pierna izquierda y la probabilidad de lesión en pierna derecha tuvo una correlación $p<0,00$ r 0,732. Tal y como se observa en el gráfico de dispersión de la Figura 1. 


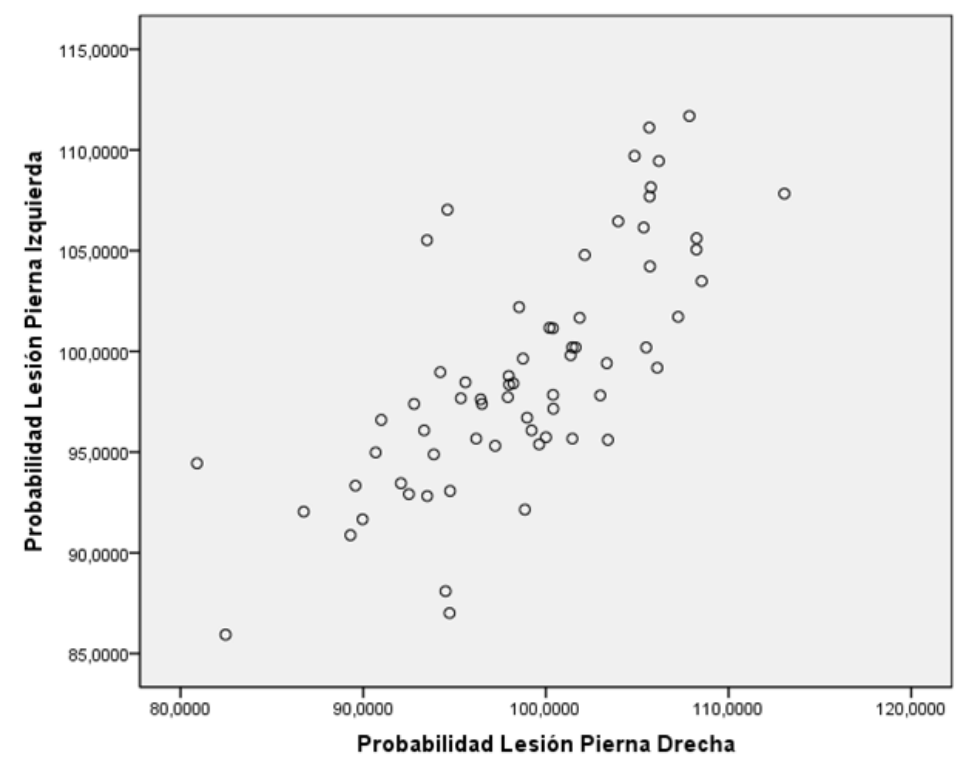

Figura 7. Gráfico de dispersión de la probabilidad de lesión en pierna izquierda y derecha.

\section{Discusión}

Este estudio analiza la probabilidad de lesión en tren inferior y porcentaje de grasa corporal en estudiantes universitarios participantes de talleres deportivos. Para medir la probabilidad de lesión se utilizó el instrumento de equilibrio YBT, mientras que, para medir el porcentaje de grasa corporal se utilizó un cáliper Slim Guide, siguiendo el protocolo Isak. El estudio de Coto, Rojas, Ortíz y Rojas (2017) busca comparar el IMC (índice de masa corporal), porcentaje de grasa y masa muscular con la prevalencia de lesiones músculo esqueléticas, a diferencia de este estudio, ellos para medir el porcentaje de grasa lo hicieron mediante bioimpedancia eléctrica y para evaluar la prevalencia de lesiones lo hicieron mediante un cuestionario elaborado tipo Likert. Su población fue de 139 varones de 20 a 35 años de edad, obteniendo como resultado una correlación positiva entre el IMC y el nivel de prevalencia de lesiones músculoesqueléticas, no obstante, no hubo correlación positiva entre el porcentaje de grasa y la probabilidad de lesión, al igual que en la presente investigación. Continuando esta línea, el estudio de Dane, Can, y Karsan (2002) investigaron atletas lesionados y no lesionados durante cuatro meses, buscando relación entre el IMC, porcentaje de grasa y fuerza en diversos músculos, el estudio arrojó como resultados que la mayor cantidad de atletas lesionados son en base a su IMC frente a los no lesionados, pero no hubo diferencia con el porcentaje de grasa corporal entre los atletas lesionados y no lesionados. Sin embargo, Jespersen et al. (2014) al estudiar lesiones en extremidades inferiores en niños considerando el IMC y porcentaje de grasa, concluyen que el riesgo de lesión aumenta cuando existe sobrepeso, pero que este factor aumenta cuando el sobrepeso es causado por un porcentaje de grasa elevado frente al de IMC.

Asimismo, el estudio de Richmond, Kang,y Emery (2013), basándose en el IMC concluye que el riesgo de sufrir una lesión es mayor en adolescentes obesos, sobre todo en los adolescentes con sobrepeso que practican 
deporte irregularmente frente a quienes tienen un nivel deportivo más alto. En este caso las lesiones fueron informadas tras haber ocurrido en el último año, después de haber encuestado a 4339 participantes.

Así también, concluyen los autores Cardozo, Cuervo-Guzman y Murcia- Torres (2016) que un IMC elevado representado principalmente por un aumento de grasa corporal es un factor de riesgo influyente en el rendimiento deportivo, reflejado en factores de riesgo para la salud.

Por otra parte, la presente investigación indica que, al existir probabilidad de lesión en una pierna, hay una fuerte correlación de que se presente en lesión en la otra. Así también lo mencionan Fernández, Rivas, y Torrens (1992) en su estudio de lesiones sobre bailarines de ballet, indicando que al momento de una lesión se afecta frecuentemente en primera instancia el miembro inferior más débil, como la pierna izquierda por ejemplo, pero con el tiempo las lesiones son más graves en el miembro contrario, o sea, en la pierna derecha. Por otra parte, la revisión sistemática elaborada por Peñalver (2018) indica en su conclusión que no existe un claro consenso entre la existencia de asimetría y aumento de riesgo de lesión.

\section{Conclusión}

Según los resultados obtenidos en esta investigación, el porcentaje de grasa tiene una leve significancia ante la probabilidad de lesión del tren inferior, por lo tanto, se puede inferir a que esta no influye en el factor lesivo de deportistas universitarios. Por otro lado, y basándonos en la discusión, se puede rescatar que el IMC si es un factor importante al momento de la lesión de tren inferior.

Las lesiones deportivas pueden ocurrir en cualquier momento y son un riesgo que corren todas las personas que realizan deporte, sin embargo, si los practicantes realizan actividad física constantemente, - realizan entrenamientos preventivos o de fortalecimiento muscular, el riesgo de lesionarse disminuye. Por tal motivo, las universidades deben contar con instalaciones e implementación deportiva adecuada para sus entrenamientos, debido a que los estudiantes universitarios no siempre cuentan con un estado físico adecuado para practicar su deporte y a la vez enfrentar la exigencia a la que exponen su cuerpo, de tal forma, disminuye el riesgo de lesionarse y de mantenerse inactivo.

Se recomienda continuar con investigaciones sobre el tema de este estudio en una mayor población y observar si existen diferentes resultados entre damas y varones, así también, observar los resultados en diferentes deportes por separado.

\section{REFERENCIAS BIBLIOGRÁFICAS}

Alvero, J., Correas, L., Ronconi, L., Fernández, R., \& Porta, J. (2011). La bioimpedancia eléctrica como método de estimación de la composición corporal, normas prácticas de utilización. Revista Andaluza de Medicina del Deporte, 4(4), 167-174. Recuperado de http://www.elsevier. es/es-revista-revista-andaluzamedicina-del-deporte-284-articulola-bioimpedancia-electrica-comometodo-X1888754611937896

Amaral, T. F., Teresa Restivo, M., Guerra, R. S., Marques, E., Chousal, M. F., \& Mota, J. (2011). Accuracy of a digital skinfold system for measuring skinfold thickness and estimating body fat. British Journal of Nutrition, 105(3), 478-484. Recuperado de https://doi. org/10.1017/s0007114510003727 
Andrade Riquelme, C., \& Villena Rodriguez, P. (2006). Estudio sobre la aplicación del "Star Excursion Balance Test" como método de entrenamiento del equilibrio dinámico y propiocepción en sujetos que presenten inestabilidad funcional de tobillo (tesis de pregado). Recuperado de http://repositorio.uchile.cl/ handle/2250/110647

Boechat de Oliveira, F., Morgado Pereira, M. D., de Mendonça Nunes, A., Barreto, R., Pecorone Matos, F., \& Gomes de Souza Vale, R. (2017). Incidencia de lesiones en la práctica deportiva del motocross. Revista Ciencias de La Actividad Física, 18(2), 1-9. Recuperado de https://doi.org/10.29035/rcaf.18.2.7

Cardozo, L. A., Cuervo Guzman, Y. A., \& Murcia Torres, J. A. (2016). Porcentaje de grasa corporal y prevalencia de sobrepeso-obesidad en estudiantes universitarios de rendimiento deportivo de Bogotá, Colombia. Nutrición Clínica Dietética y Hospitalaria, 36(3), 68-75. DOl: 10.12873/363cardozo

Chimera, N. J., Smith, C. A., \& Warren, M. (2015). Injury history, sex, and performance on the functional movement screen and $Y$ balance test. Journal of Athletic Training, 50(5), 475-485. DOl: 10.4085/1062-6050-49.6.02

Coto Cordero, D., Rojas Quirós, J., Ortíz Ulloa, J., \& Rojas Gómez, J. M. (2017). Relación del índice de masa corporal (imc), porcentajes de grasa y de masa muscular, con el nivel de prevalencia de lesiones músculo-esqueléticas en hombres de los 20 a los 35 años. Revista Académica Educar, 7(1), 1-14. Recuperado de https://www.uca. ac.cr/wp-content/uploads/2017/09/ Revista-Educar-V1N10617A1-7.pdf
Dane, Ş., Can, S., \& Karsan, O. (2002). Relations of Body Mass Index, Body Fat, and Power of Various Muscles to Sport Injuries. Perceptual and Motor Skills, 95(1), 329-334. Recuperado de https:// doi.org/10.2466/pms.2002.95.1.329

Fernández-Palazzi, F., Rivas Hernández, S., \& Torrens, D. P. (1992). Lesiones en bailarines de Ballet Clásico (Estudio estadístico de cuatro años). Archivos de Medicina del Deporte 9(35), 309313 Recuperado de http://femede.es/ documentos/Ballet_309_35.pdf

Gribble, P. A., Hertel, J., \& Plisky, P. (2012). Using the Star Excursion Balance Test to Assess Dynamic PosturalControl Deficits and Outcomes in Lower Extremity Injury: A Literature and Systematic Review. Journal of Athletic Training, 47(3), 339-357. DOI: 10.4085/1062-6050-47.3.08

Hewitt, G. B., Withers, R. T., Brooks, A. G., Woods, G. F., \& Day, L. A. (2002). Improved rig for dynamically calibrating skinfold calipers: Comparison between Harpenden and Slim Guide instruments. American Journal of Human Biology, 74(6), 721-727. https://doi.org/10.1002/ ajhb.10087

Jespersen, E., Verhagen, E., Holst, R., Klakk, H., Heidemann, M., Rexen, C. T., ... Wedderkopp, N. (2014). Total body fat percentage and body mass index and the association with lower extremity injuries in children: a 2.5-year longitudinal study. British Journal of Sports Medicine, 48(20), 1497-1502. Recuperado de https://doi. org/10.1136/bjsports-2013-092790

Kenney, W. L., Wilmore, J. H., Costill, D. L., \& Wilmore, J. H. (2012). Physiology of sport and exercise. Champaing, IL: Human Kinetics, Inc. 
Llacer, A. (2015). Efectividad de los diferentes medios utilizados para evaluar la inestabilidad del tobillo. Trabajo de fin de grado opción: revisión bibliográfica. Universidad Miguel Hernández de Elche, España. Recuperado de http://dspace.umh. es/handle/11000/2505

Martín, A., Ross, W., Drinkwater, D., \& Clarys, J. (1992). Predicción sobre tejido adiposo corporal, mediante técnica de calibre para pliegues cutáneos: suposiciones y evidencia cadavérica. PublicE, 7. Recuperado de https://g-se.com/ prediccion-sobre-tejido-adiposocorporal-mediante-tecnica-decalibre-para-pliegues-cutaneossuposiciones-y-evidenciacadaverica-221-sa-O57cfb2711967d

Martínez, S., Pasquarelli, B. N., Romaguera, D., Arasa, C., Tauler, P., \& Aguiló, A. (2011). Anthropometric Characteristics and Nutritional Profile of Young Amateur Swimmers. Journal of Strength and Conditioning Research, 25(4), 1126-1133. Recuperado de https://doi. org/10.1519/JSC.0b013e3181d4d3df

Medina, D., Lizarraga, A., \& Drobnic, F. (2014). Injury prevention and nutrition in football. Sports Science Exchange, 27(132), 1-5. Recuperado de https:// secure.footprint.net/gatorade/stg/ gssiweb/pdf/SSE132_Medina.pdf

Meeuwisse, W. H., Tyreman, H., Hagel, B., \& Emery, C. (2007). A dynamic model of etiology in sport injury: the recursive nature of risk and causation. Clinical Journal of Sport Medicine, 77(3), 215-219. Recuperado de https://doi. org/10.1097/JSM.0b013e3180592a48
Moreno, V. M., Gómez Gandoy, J. B., \& Antoranz González, M. J. (2001). Medicíon de la grasa corporal mediante impedancia bioeléctrica, pliegues cutáneos y ecuaciones a partir de medidas antropométricas. Análisis comparativo. Revista Española de Salud Pública, 75(3), 221-236. Recuperado de https://doi.org/10.1590/s113557272001000300006

Osorio Ciro, J. A., Clavijo Rodríguez, M. P., Arango V., E., Patiño Giraldo, S., \& Gallego Ching, I. C. (2007). Lesiones deportivas. latreia, 20(2), 167-177. Recuperado de https://www.redalyc. org/articulo.oa?id=180513859006

Padilla, J., Taylor, A., Yuhasz, M., \& Velazquez, M. (2004). Algunas características antropométricas de una población de atletas mexicanos. Revista Médica Del Hospital General de México, 67(1), 11-21. Recuperado de https://www. medigraphic.com/pdfs/h-gral/hg2004/hg041c.pdf

Peñalver Navarro, D. (2018). Relación entre asimetrías y lesiones en el deporte: una revisión sistemática. (Tesis de pregrado) Universidad de Valencia, España.

Plisky, P. J., Gorman, P. P., Butler, R. J., Kiesel, K. B., Underwood, F. B., \& Elkins, B. (2009). The reliability of an instrumented device for measuring components of the star excursion balance test. North American Journal of Sports Physical Therapy: NAJSPT, 4(2), 9299. Recuperado de http://www.ncbi. nlm.nih.gov/pubmed/21509114

Richmond, S. A., Kang, J., \& Emery, C. A. (2013). Is body mass index a risk factor for sport injury in adolescents? Journal of Science and Medicine in Sport, 16(5), 401-405. Recuperado de https:// doi.org/10.1016/J.JSAMS.2012.11.898 
Rivera, J. (2002). Caracterización del perfil antropométrico del atleta universitario de 1998 (Tesis de maestría). Universidad Autónoma de Chihuahua, México. Recuperado de http://www.universiada.uach. mx/universiada2010/noticias/ PROTOCOLO\%2OUNIVERSIADA\%20 2010.pdf

Stevenson, M. R., Hamer, P., Finch, C. F., Elliot, B., \& Kresnow, M. (2000). Sport, age, and sex specific incidence of sports injuries in Western Australia. British Journal of Sports Medicine, 34(3), 188-194. Recuperado de http://www. ncbi.nlm.nih.gov/pubmed/10854018
Stewart, A., Marfell-Jones, M., Olds, T., \& de Ridder, H. (2011). Protocolo internacional para la valoración antropométrica. ISAK. International Society for the Advancement of Kinanthropometry. Recuperado de https://es.scribd.com/ document/375628061/ProtocoloAntropometria-2011-ISAK

Warburton, D. E. R., Nicol, C. W., \& Bredin, S. S. D. (2006). Health benefits of physical activity: the evidence. Canadian Medical Association Journal, 774(6), 801-809. Recuperado de https://doi. org/10.1503/cmaj.051351

\section{Dirección para correspondencia}

Vanessa Carrasco Alarcón

Doctora en Ciencias de la Motricidad Humana. Departamento de Educación Física, Deportesy Recreación. Universidad de La Frontera.

Temuco, Chile.

ORCID: https://orcid.org/0000-0002-2713-4506

Contacto:

vanessa.carrasco@ufrontera.cl

Recibido: 14-09-2019

Aceptado: 04-05-2020 